\title{
Letter \\ UV Irradiation-Induced SERS Enhancement in Randomly Distributed Au Nanostructures
}

\author{
Dong-Jin Lee ${ }^{1}(\mathbb{D})$ and Dae Yu Kim ${ }^{1,2, *}$ \\ 1 Inha Research Institute for Aerospace Medicine, Inha University, Incheon 22212, Korea; \\ voinaimir82@gmail.com \\ 2 Department of Electrical Engineering, College of Engineering, Inha University, Incheon 22212, Korea \\ * Correspondence: dyukim@inha.ac.kr; Tel.: +82-32-860-7394
}

Received: 8 June 2020; Accepted: 8 July 2020; Published: 9 July 2020

\begin{abstract}
Currently used platforms for surface-enhanced Raman scattering (SERS) sensors generally employ metallic nanostructures for enrichment of the plasmonic hotspots in order to provide higher Raman signals, but this procedure is still considered challenging for analyte-surface affinity. This study reports a UV irradiation-induced SERS enhancement that amplifies the interactions between the analytes and metallic surfaces. The UV light can play critical roles in the surface cleaning to improve the SERS signal by removing the impurities from the surfaces and the formation of the negatively charged adsorbed oxygen species on the Au surfaces to enhance the analyte-surface affinity. To evaluate this scenario, we prepared randomly distributed Au nanostructures via thermal annealing with a sputtered Au thin film. The UV light of central wavelength $254 \mathrm{~nm}$ was then irradiated on the Au nanostructures for $60 \mathrm{~min}$. The SERS efficiency of the Au nanostructures was subsequently evaluated using rhodamine $6 \mathrm{G}$ molecules as the representative Raman probe material. The Raman signal of the Au nanostructures after UV treatment was enhanced by up to approximately $68.7 \%$ compared to that of those that did not receive the UV treatment. We expect that the proposed method has the potential to be applied to SERS enhancement with various plasmonic platforms.
\end{abstract}

Keywords: surface-enhanced Raman scattering; UV treatment; negatively charged adsorbed oxygen species; analyte-surface affinity; Au nanostructures

\section{Introduction}

Boosting the Raman signals in surface-enhanced Raman scattering (SERS) sensors has been extensively studied for decades, and the SERS sensors typically employ metallic nanostructures that generate plasmonic hotspots to concentrate the localized electromagnetic fields in the vicinity of the plasmonic nanostructures [1-5]. Various metallic nanostructures have been developed thus far for amplifying the plasmonic hotspots, including low-dimensional metallic nanostructures with highly localized electromagnetic field and two-/three-dimensional architectures with increased surface areas [5-11]. In addition, new analytical tools, including tip-enhanced Raman spectroscopy and shell-isolated nanoparticle-enhanced Raman spectroscopy, have been proposed for single-molecule detection with spatial resolution on the order of the sub-diffraction limit [5,12-16].

Despite extensive studies on hotspot engineering, a majority of the current SERS platforms rarely consider the effects of analyte adsorption on the metallic surfaces $[2,17,18]$. Poor analyte affinities to the metallic surfaces are quite problematic, because a high-performance SERS platform can be useless for detecting the analytes that do not interact with the electromagnetic hotspots. This remains a formidable issue for detecting analytes that have no interactions with metallic surfaces [2]. Therefore, strategies for enhancing the analyte-surface affinities are required for greater adoption of SERS sensors for practical applications. So far, four main methodologies have been presented to enhance the analyte affinities to 
the metallic surfaces, including chemical interactions between the analytes and the metallic surfaces, physical confinement of the analytes close to the metallic surfaces, encapsulation of the metallic surfaces for the concentration of the analytes on the electromagnetic hotspots, and chemical modifications of the analyte molecules [2]. Among them, the surface charges generated on the plasmonic surfaces can play a critical role for enhancing the analyte-surface affinities to promote the adsorption of polar as well as non-polar molecules [19-21].

UV-ozone treatment is an effective, simple, and powerful tool for surface modification and activation, which generates the reactive oxygen species on the film surfaces [22-26]. UV-ozone treatment cleans the metallic surfaces to improve the SERS signal by removing the impurities on the surfaces, facilitating the adsorption of analyte molecules [27-29]. Furthermore, UV-ozone treatment can be expected to induce the negatively charged adsorbed oxygen species on the Au surfaces. Saliba et al. reported that the electrons transfer from the Au surface into the adsorbed oxygen layer [30], and Sun et al. theoretically studied the relation between the adsorbed oxygen atoms and the Au surface atoms using the Hückel theory and density functional theory (DFT) calculations [31]. In addition, Hai et al. reported the formation of the surface charges on the Au surface due to the oxygen adsorption [20], and these negatively charged adsorbed oxygen species could play a role in the enhancement of the analyte-surface affinities [21]. Therefore, UV-ozone exposure can be used to boost the SERS spectra by the UV-ozone cleaning of the surface and the UV-ozone induced negatively charged adsorbed oxygen species.

In this work, we present an alternative strategy for SERS enhancement utilizing UV irradiation-induced surface cleaning and negatively charged adsorbed oxygen species for amplifying the interactions between the analytes and metallic surfaces. To this end, we prepared randomly distributed $\mathrm{Au}$ nanostructures via thermal annealing with a sputtered $\mathrm{Au}$ thin film. The UV light of central wavelength $254 \mathrm{~nm}$ was irradiated on the Au nanostructures for $60 \mathrm{~min}$ to enhance the analyte-surface affinity. The SERS enhancement was measured using rhodamine 6G (R6G) molecules as the representative Raman probe material, and a Raman signal increase of up to approximately $68.7 \%$ was observed. The proposed method therefore has the potential to be applied to SERS enhancement on various plasmonic platforms.

\section{Experimental Procedures}

\subsection{Materials}

Silicon wafers were purchased from Silicon Materials Inc., USA (B-doped p-type wafer, resistivity of 1-30 $\Omega \cdot \mathrm{cm})$. Rhodamine 6G (R6G) was purchased from Sigma-Aldrich Inc., South Korea; the Au target was purchased from Thifine Inc., Incheon, South Korea (2-inch diameter, purity of 99.99\%).

\subsection{Fabrication}

The silicon wafers were cleaned using acetone, isopropyl alcohol, and deionized water in an ultrasonication bath. Then, $\mathrm{Au}$ thin films of thickness 3.4, 4.5, and $6.4 \mathrm{~nm}$ were deposited on the Si wafers via direct current (DC) sputtering with a base pressure of $5 \times 10^{-6}$ Torr. A constant DC voltage of $350 \mathrm{~V}$ was applied, and argon was used to produce the plasma with $10 \mathrm{sccm}$. To fabricate the randomly distributed $\mathrm{Au}$ nanostructures, the $\mathrm{Au}$ thin film was thermally annealed on a hotplate at temperatures of 150,250 , and $350{ }^{\circ} \mathrm{C}$ under ambient conditions for $60 \mathrm{~min}$. The samples were cooled freely in ambient conditions, and a UV lamp $(2 \times 15 \mathrm{~W}$, UVITEC, Cambridge, UK) generating light with a central wavelength of $254 \mathrm{~nm}$ was used to irradiate the randomly distributed Au nanostructures for $60 \mathrm{~min}$. After UV treatment, the Au nanostructures were soaked in rhodamine 6G aqueous solution of concentration $1 \mathrm{M}$ for $60 \mathrm{~min}$. 


\subsection{Characterization and Measurements}

The morphologies of the randomly distributed Au nanostructures were characterized using scanning electron microscopy (SEM; Hitachi S-4300SE, Hitachi, Tokyo, Japan) and atomic force microscopy (AFM; NanoScope IV, Bruker, MA, USA). Raman spectra were measured via Raman spectroscopy (LabRAM HR Evolution, HORIBA, Kyoto, Japan) using an excitation wavelength of $532 \mathrm{~nm}$, a laser power of $2 \mathrm{~mW}$, an acquisition time of $5 \mathrm{~s}$, and an accumulation of 1 . The Raman spectra were obtained in the range of $400-1800 \mathrm{~cm}^{-1}$ for all experiments.

\section{Results and Discussion}

Figure 1a presents a schematic of the fabrication process for the randomly distributed Au nanostructures. The Au thin film was deposited via DC sputtering by adjusting the deposition time to control Au film thickness. The Au thin film was then aggregated into different sizes and shapes through thermal annealing. The UV light with a central wavelength of $254 \mathrm{~nm}$ was irradiated on the Au thin film for $60 \mathrm{~min}$ to fabricate the randomly distributed Au nanostructures. The SERS performances of the Au nanostructures irradiated with or without UV light were evaluated using R6G molecules adsorbed on the Au nanostructures, as shown in Figure $1 \mathrm{~b}$.

(a)
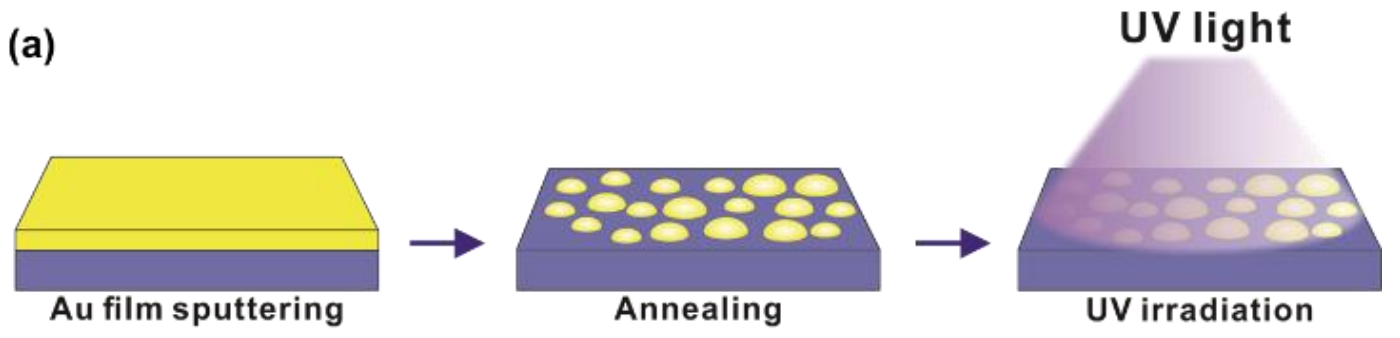

(b)
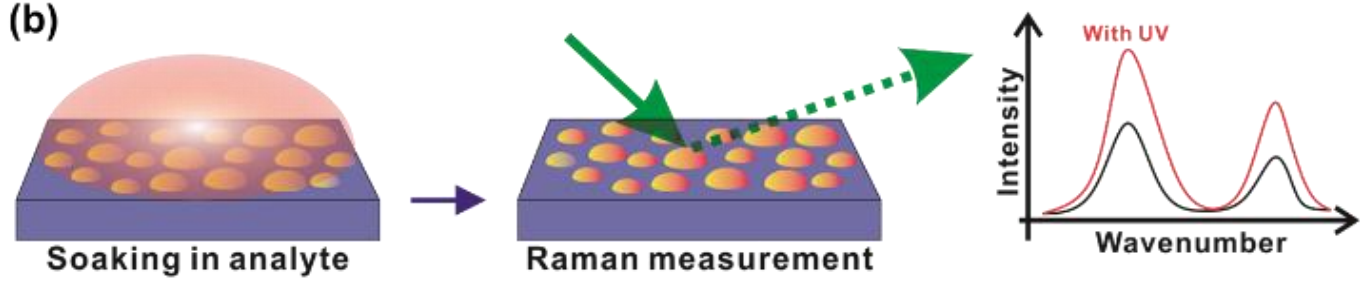

Figure 1. Schematic of UV irradiation-induced surface-enhanced Raman scattering (SERS) enhancement: (a) fabrication of the randomly distributed Au nanostructures by thin film sputtering, thermal annealing, and UV treatments; (b) SERS performance of the Au nanostructures irradiated with or without UV light and evaluated using rhodamine 6G (R6G) molecules as the Raman probe material.

Figure 2 shows the SEM and AFM images of the randomly distributed Au nanostructures depicting the effect of initial thickness $(3.4,4.5$, and $6.4 \mathrm{~nm})$ and annealing temperature $\left(150,250\right.$, and $\left.350{ }^{\circ} \mathrm{C}\right)$. The film thickness was determined from the cross-sectional profile of the film on a Si substrate after scratching a section of Au thin film (see Figure A1 in Appendix A.1). Uniformly distributed Au nanograins were observed for samples of Au nanostructures annealed at room temperature (RT). The root mean-squared (RMS) roughness value for Au3.4, Au4.5, and Au6.4 samples at RT were $0.1835 \mathrm{~nm}, 0.2264 \mathrm{~nm}$, and $0.25 \mathrm{~nm}$, respectively. With an increase in the annealing temperature, the grain sizes of the Au nanostructures increased. For the Au3.4 sample, the RMS roughness increased from $0.1835 \mathrm{~nm}$ at RT annealing to $0.43 \mathrm{~nm}$ at $350{ }^{\circ} \mathrm{C}$ annealing. For the Au4.5 sample, the RMS roughness increased from $0.2264 \mathrm{~nm}$ at $\mathrm{RT}$ annealing to $0.9979 \mathrm{~nm}$ at $350{ }^{\circ} \mathrm{C}$ annealing. For the Au6.4 sample, the RMS roughness increased from $0.25 \mathrm{~nm}$ at RT annealing to $9.329 \mathrm{~nm}$ at $350{ }^{\circ} \mathrm{C}$ annealing. In addition, it was observed that in the case of Au nanostructures having large initial thickness (Au6.4), the grain size and RMS roughness rapidly increased (for more detailed information, see Figure A2 in Appendix A.2). 




Figure 2. SEM and atomic force microscopy (AFM) images of randomly distributed Au nanostructures, showing the grain size variation of Au nanostructures with annealing temperature and initial $\mathrm{Au}$ film thickness. Insets: The size of AFM images are all $1 \times 1 \mu \mathrm{m}^{2}$.

Prior to evaluation of the SERS performances of the Au nanostructure by UV treatment, the Raman spectra depending on annealing temperatures and initial thicknesses were investigated in order to elucidate the SERS effect for these Au nanostructures. Figure 3a shows the Raman spectrum of the Au4.5 sample annealed at RT. This spectrum shows strong peaks of the vibrational bands at approximately $611,773,1310,1363$, and $1651 \mathrm{~cm}^{-1}$, corresponding to the Raman characteristic peaks of R6G [32-34]. The vibrational band at $611 \mathrm{~cm}^{-1}$ is due to the in-plane and out-of-plane xanthene ring deformations. The vibrational band at $773 \mathrm{~cm}^{-1}$ is due to the out-of-plane $\mathrm{C}-\mathrm{H}$ bending and in-plane xanthene ring deformations. The vibrational band at $1310 \mathrm{~cm}^{-1}$ is due to the in-plane xanthene ring breath and in-plane N-H bending. The vibrational bands at 1363 and $1651 \mathrm{~cm}^{-1}$ originated from the xanthene ring stretching and in-plane $\mathrm{C}-\mathrm{H}$ bending. The Raman peak of around $611 \mathrm{~cm}^{-1}$ is the most dominant R6G characteristic peak, and we selected this peak for comparison of the SERS activities of the randomly distributed Au nanostructures. Figure $3 \mathrm{~b}$ shows the Raman signals of the Au3.4, Au4.5, and Au6.4 samples at $611 \mathrm{~cm}^{-1}$, depending on the annealing temperatures. A film formed by the evaporation process grows according to the following four stages: nucleation, growth of the nuclei and formation of larger islands, coalescence of the islands and formation of a connected network containing empty channels, and filling of the channels [35]. According to the aforementioned film-growth evolution, it can be expected that the plasmonic hotspots may diminish as the Au initial film thickness increases, thereby causing the SERS performance to deteriorate. For RT annealing, the Raman signals of the Au3.4 and Au4.5 samples are approximately twice that of the Au6.4 sample, presumably owing to the decrease in plasmonic hotspots. With the increase in annealing temperature, the Raman signals show a decreasing trend for the Au3.4, Au4.5, and Au6.4 samples, owing to gap widening between the Au nanostructures, thus diminishing the plasmonic hotspots. 



Figure 3. Raman spectra for $1 \mu \mathrm{M}$ of R6G adsorbed on the randomly distributed Au nanostructures. (a) Raman spectrum of the Au4.5 sample annealed at RT. (b) Raman signals of the Au3.4, Au4.5, and Au6.4 samples at $611 \mathrm{~cm}^{-1}$ as a function of the annealing temperatures.

To investigate the SERS performances of the Au nanostructures after UV treatment, the Raman signals from 10 samples of the Au nanostructures were compared as a function of UV treatment, as depicted in Figure 4. The Raman signals generally increased in the UV-treated Au nanostructures compared to the UV-untreated Au nanostructures. The SERS enhancement in each case was calculated as $\left(I_{U V}-I_{0}\right) / I_{0}(\%)$, where $I_{U V}$ and $I_{0}$ are the Raman intensities with and without UV treatment, respectively. Table 1 shows the Raman signal enhancement of Au nanostructures before and after UV treatment. The Raman signal of the Au4.5 sample annealed at $350{ }^{\circ} \mathrm{C}$ increased to about $68.75 \%$ after UV treatment, showing the greatest Raman signal enhancement. The SEM and AFM images were also compared to investigate the morphological variations of the Au nanostructures from UV treatment. As illustrated in Figure A3 in Appendix A.3, the morphological changes of the Au nanostructures with and without UV irradiation were not readily revealed. The RMS roughness values of the Au4.5 sample with and without UV treatment were about $1.745 \mathrm{~nm}$ and $1.776 \mathrm{~nm}$, respectively.

(a)

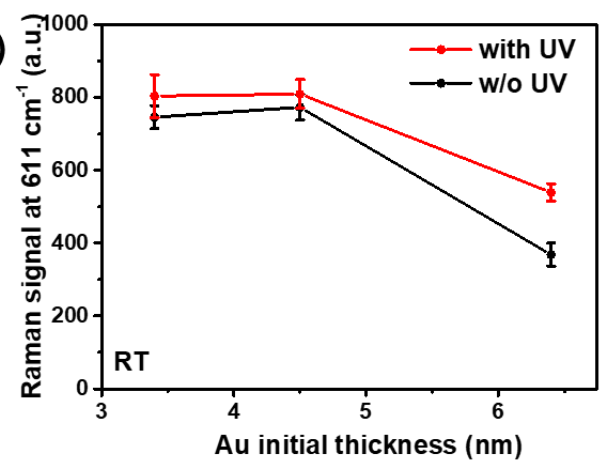

(c)

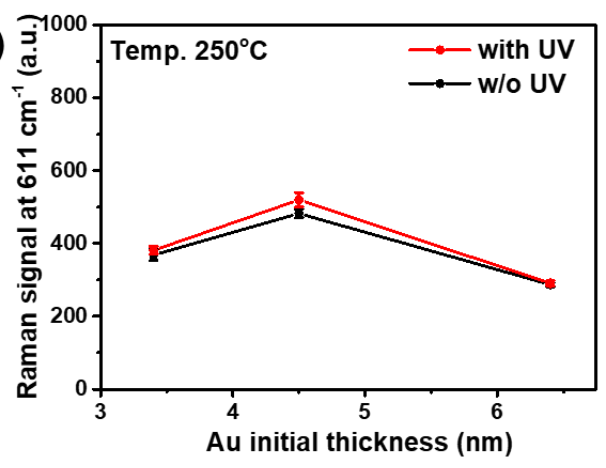

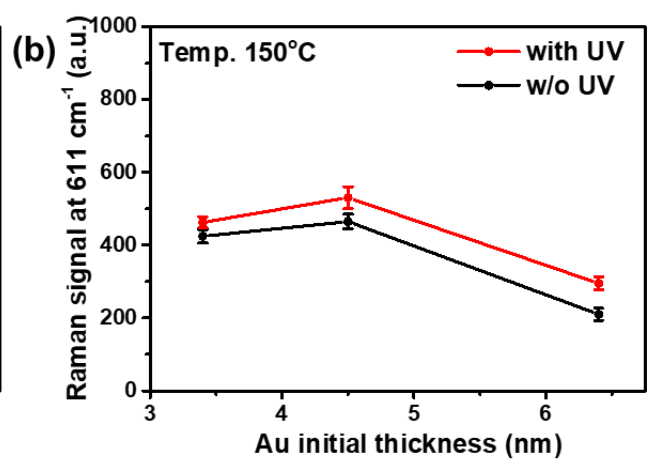

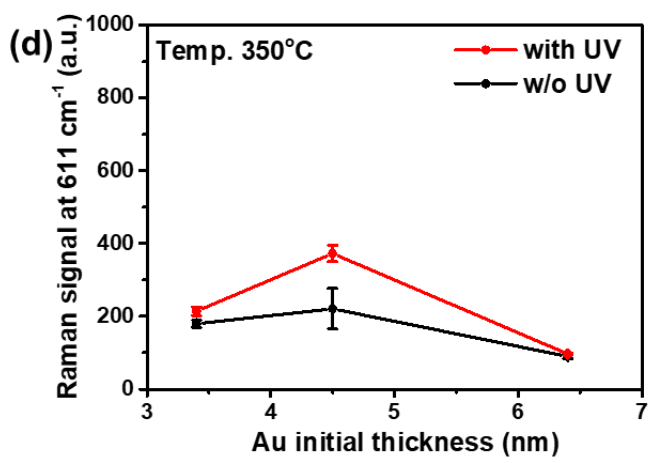

Figure 4. Raman signals of the Au3.4, Au4.5, and Au6.4 samples with and without UV treatment for annealing at (a) room temperature (RT), (b) $150{ }^{\circ} \mathrm{C}$, (c) $250^{\circ} \mathrm{C}$, and (d) $350{ }^{\circ} \mathrm{C}$. 
Table 1. SERS enhancement with UV treatment $\left(I_{U V}-I_{0}\right) / I_{0}(\%)$.

\begin{tabular}{ccccc}
\hline & $\mathbf{R T}$ & $\mathbf{1 5 0}{ }^{\circ} \mathbf{C}$ & $\mathbf{2 5 0}{ }^{\circ} \mathbf{C}$ & $\mathbf{3 5 0}{ }^{\circ} \mathbf{C}$ \\
\hline Au3.4 & $7.76 \%$ & $8.95 \%$ & $3.54 \%$ & $19.28 \%$ \\
\hline Au4.5 & $4.68 \%$ & $14.14 \%$ & $7.72 \%$ & $68.75 \%$ \\
\hline Au6.4 & $46.44 \%$ & $40.61 \%$ & $1.3 \%$ & $7.28 \%$ \\
\hline
\end{tabular}

UV-ozone treatment is expected to function as surface cleaning for the removal of the impurities on the metallic surfaces and the formation of the negatively charged adsorbed oxygen species. UV-induced surface cleaning is a well-known process, and plays a certain role of dissociating directly the $\mathrm{C}-\mathrm{C}$ and the $\mathrm{C}-\mathrm{H}$ bonds in the adsorbed organic molecules on the Au surfaces, as detailed in the literature [36-38]. This cleaning effect enables more adsorption of R6G molecules onto Au nanostructures and may contribute to the improvement of the SERS signal. In addition, the UV-induced negatively charged adsorbed oxygen species can also cause an enhancement in the SERS signal. Figure 5 shows the schematic of the mechanism of the SERS enhancement by the UV irradiation-induced negatively charged adsorbed oxygen species, thereby enabling the analyte-surface affinity. Under UV irradiation of the Au nanostructures, the excited electrons generated from the Au bulk are transferred into the adsorbed oxygen ions, generating the negatively charged adsorbed oxygen species on the surfaces of the Au nanostructures. The negatively charged Au nanostructures seem to undergo an attractive electrostatic interaction with the R6G molecules. This interaction enhances the adsorption of the R6G molecules, resulting in the enhancement of the SERS intensities.
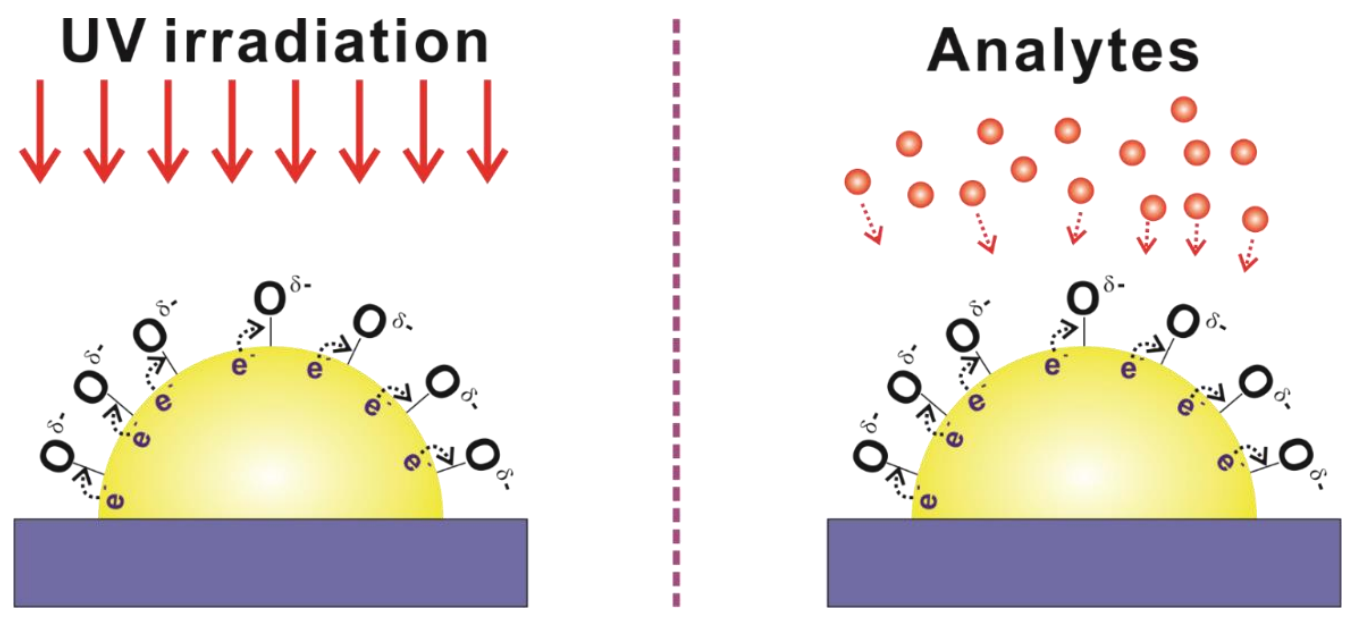

Figure 5. Schematic of the mechanism for the SERS enhancement by the UV irradiation-induced negatively charged adsorbed oxygen species. Under UV irradiation, the excited electrons from the Au bulk transfer into adsorbed oxygen ions, thus increasing the analyte-surface affinity.

\section{Conclusions}

We proposed and demonstrated a new strategy for SERS enhancement utilizing UV treatment for amplifying the interactions between analytes and metallic surfaces. The UV irradiation seems to function as surface cleaning and promote the formation of the negatively charged adsorbed oxygen species on the surfaces of Au nanostructures. These effects are expected to enhance the analyte-surface affinity and enable the SERS enhancement, but it has not been clarified yet which one is more dominant. The SERS performance using this proposed method was measured using R6G molecules, and a Raman signal increase of up to approximately $68.7 \%$ was observed. The proposed method therefore has the potential to be applied to SERS enhancement on various plasmonic platforms. 
Author Contributions: D.-J.L. fabricated the sensor devices and performed the sensing experiments. D.Y.K. and D.-J.L. drafted the manuscript. All authors have read and agreed to the published version of the manuscript.

Funding: This work was supported by the National Research Foundation of Korea (NRF), the Ministry of Education, through the Basic Science Research Program under Grant No. 2018R1A6A1A03025523, by the National Research Foundation of Korea (NRF) funded by the Ministry of Science and ICT, under Grant No. 2019R1A2C1006814 and No. 2019M3E5D1A02069623, and by the INHA UNIVERSITY Research Grant.

Conflicts of Interest: The authors declare no conflict of interest.

\section{Appendix A}

These Appendixes offers the information on the surface roughness, the film thickness, and the change of the surface morphologies before and after UV treatment of the Au nanostructures. Gwyddion v2.53, which is an open source software, was used to analyze the atomic force microscopy (AFM) images for data analysis of the surface roughness and the film thickness.

\section{Appendix A.1. Thickness of Au Thin Film}

AFM film thickness measurements were made by scratching a section of Au thin film. The film thickness was determined from the cross-sectional profile of the scratch of $\mathrm{Au}$ thin film on the $\mathrm{Si}$ substrate. Figure A1a shows the AFM image of the scratched section of Au thin film. Figure A1b depicts the cross-sectional profile of the scratched region (red line in Figure A1a as a function of the sputtering time. The average thickness and standard deviation of Au3.4 sample were $3.41 \mathrm{~nm}$ and $0.118 \mathrm{~nm}$, respectively. The average thickness and standard deviation of Au4.5 sample were $4.48 \mathrm{~nm}$ and $0.0893 \mathrm{~nm}$, respectively. The average thickness and standard deviation of Au6.4 sample were $6.42 \mathrm{~nm}$ and $0.0771 \mathrm{~nm}$, respectively.
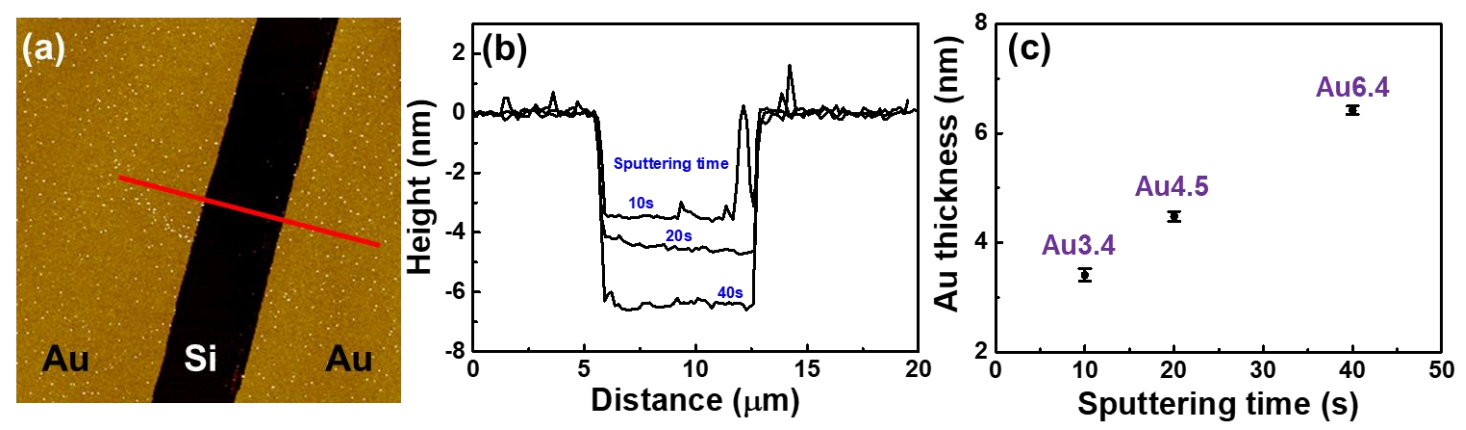

Figure A1. The thickness of Au thin film. (a) Atomic force microscopy (AFM) image of the scratched section of Au thin film. (b) Cross-sectional profile of the scratch. (c) Au thickness as a function of the sputtering time.

\section{Appendix A.2. RMS Roughness of Au Nanostructures}

The root mean square (RMS) roughness (Rq), one of the dispersion parameters for characterizing the surface roughness, was obtained by squaring each height value in the dataset, then taking the square root of the mean. Figure A2a shows the AFM image of the Au6.4 sample annealed at $350{ }^{\circ} \mathrm{C}$. Figure $\mathrm{A} 2 \mathrm{~b}$ shows the RMS roughness as a function of the annealing temperature. The RMS roughness of the Au6.4 sample rapidly increased from $0.25 \mathrm{~nm}$ at RT annealing to $9.329 \mathrm{~nm}$ at $350{ }^{\circ} \mathrm{C}$ annealing as shown in Figure A2b. 

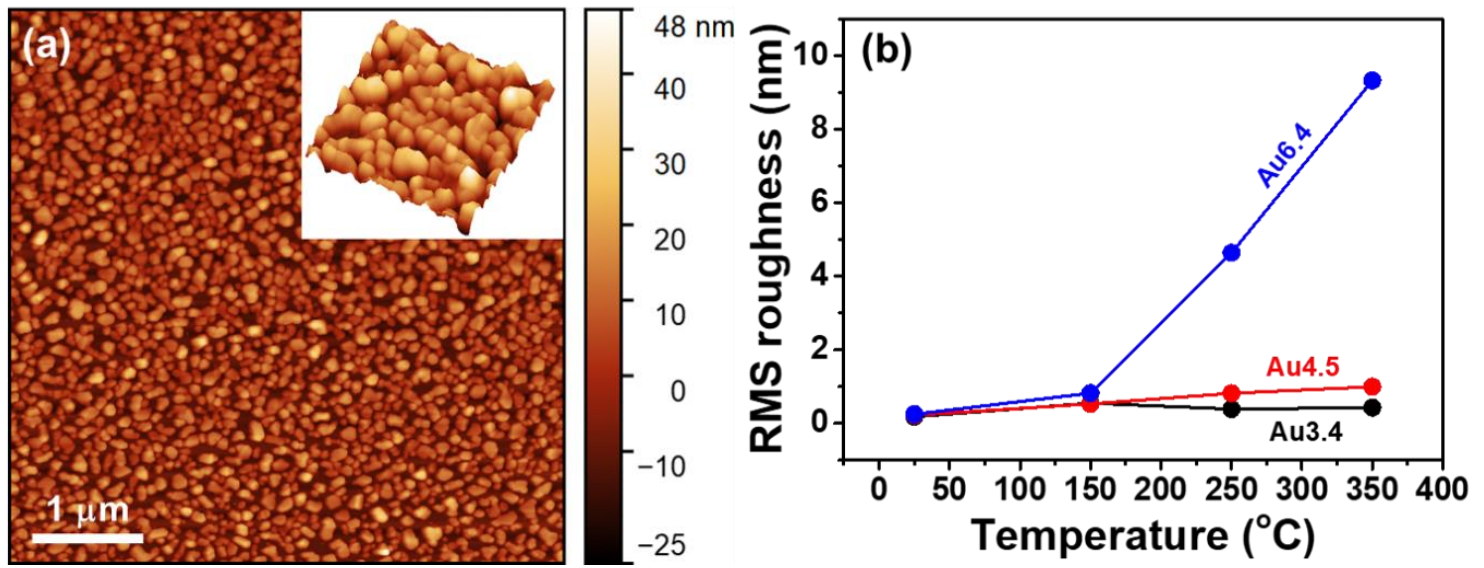

Figure A2. The root mean square (RMS) roughness of the Au3.4, Au4.5, and Au6.4 samples. (a) The AFM image of the Au6.4 sample annealed at $350{ }^{\circ} \mathrm{C}$. The $3 \mathrm{D}$ image is shown in inset. (b) The RMS roughness as a function of the annealing temperatures.

Appendix A.3. Surface Morphology Before and After UV Treatment

Figure A3 shows the SEM and AFM images before and after UV treatment. The morphological changes of the Au nanostructures with and without UV irradiation were not readily revealed.
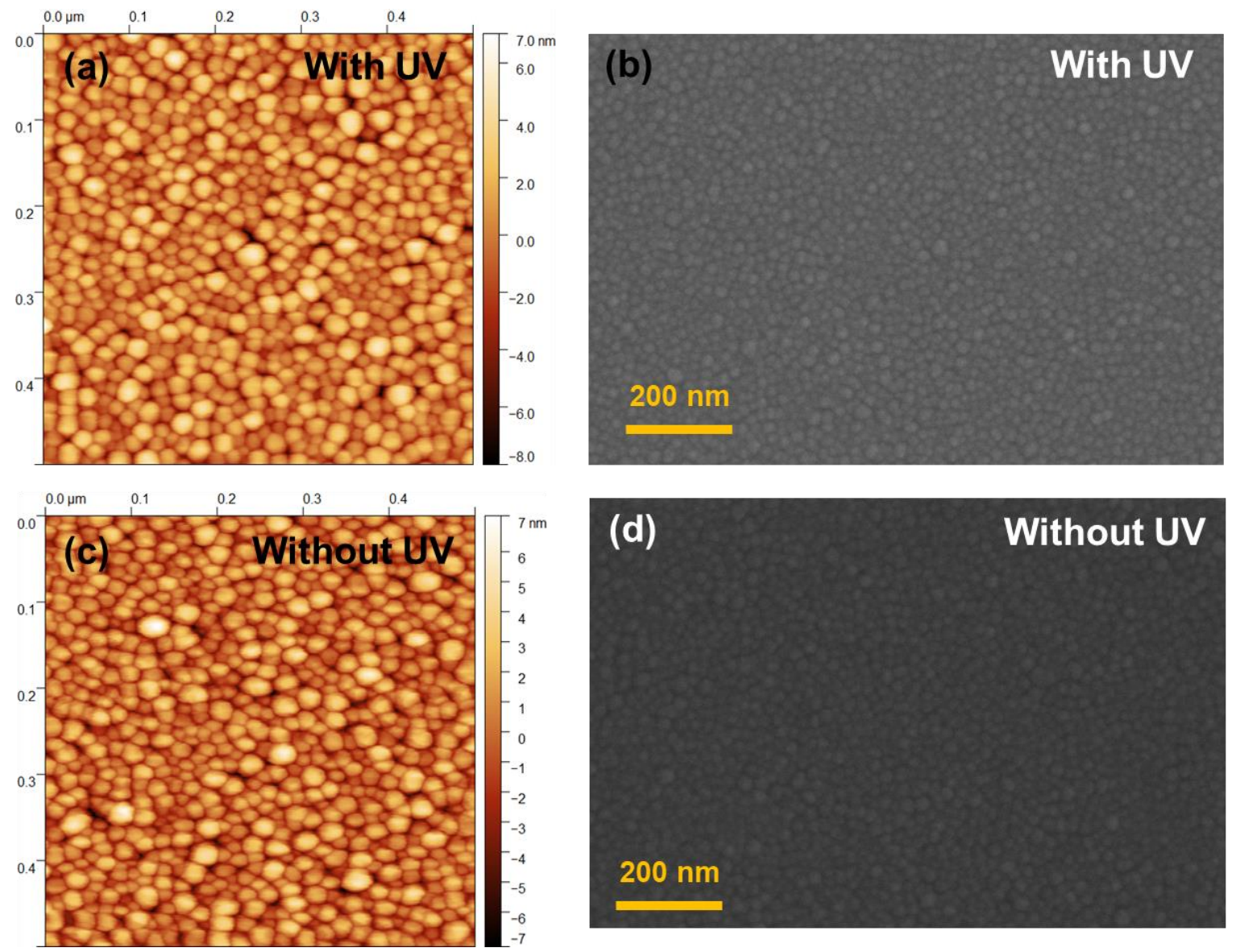

Figure A3. (a-d) AFM and SEM images of the Au4.5 sample annealed at $350{ }^{\circ} \mathrm{C}$ before and after UV treatment. The size of AFM images are all $500 \times 500 \mathrm{~nm}^{2}$. 


\section{References}

1. Langer, J.; Jimenez de Aberasturi, D.; Aizpurua, J.; Alvarez-Puebla, R.A.; Auguie, B.; Baumberg, J.J.; Bazan, G.C.; Bell, S.E.J.; Boisen, A.; Brolo, A.G.; et al. Present and Future of Surface-Enhanced Raman Scattering. ACS Nano 2020, 14, 28-117. [CrossRef] [PubMed]

2. Lee, H.K.; Lee, Y.H.; Koh, C.S.L.; Gia, C.P.Q.; Han, X.M.; Lay, C.L.; Sim, H.Y.F.; Kao, Y.C.; An, Q.; Ling, X.Y. Designing surface-enhanced Raman scattering (SERS) platforms beyond hotspot engineering: Emerging opportunities in analyte manipulations and hybrid materials. Chem. Soc. Rev. 2019, 48, 731-756. [CrossRef] [PubMed]

3. Li, J.F.; Zhang, Y.J.; Ding, S.Y.; Panneerselvam, R.; Tian, Z.Q. Core-Shell Nanoparticle-Enhanced Raman Spectroscopy. Chem. Rev. 2017, 117, 5002-5069. [CrossRef] [PubMed]

4. Ding, S.Y.; You, E.M.; Tian, Z.Q.; Moskovits, M. Electromagnetic theories of surface-enhanced Raman spectroscopy. Chem. Soc. Rev. 2017, 46, 4042-4076. [CrossRef]

5. Ding, S.Y.; Yi, J.; Li, J.F.; Ren, B.; Wu, D.Y.; Panneerselvam, R.; Tian, Z.Q. Nanostructure-based plasmon-enhanced Raman spectroscopy for surface analysis of materials. Nat. Rev. Mater. 2016, 1, 16021. [CrossRef]

6. Tang, L.; Li, S.; Han, F.; Liu, L.; Xu, L.; Ma, W.; Kuang, H.; Li, A.; Wang, L.; Xu, C. SERS-active Au@Ag nanorod dimers for ultrasensitive dopamine detection. Biosens. Bioelectron. 2015, 71, 7-12. [CrossRef]

7. Ma, W.; Kuang, H.; Wang, L.; Xu, L.; Chang, W.S.; Zhang, H.; Sun, M.; Zhu, Y.; Zhao, Y.; Liu, L.; et al. Chiral plasmonics of self-assembled nanorod dimers. Sci. Rep. 2013, 3, 1934. [CrossRef]

8. Ahmed, I.; Abdelrahman, A.M.M. Takeyoshi Okajima, and Takeo Ohsaka, Fabrication and Electrochemical Application of Three-Dimensional Gold Nanoparticles: Self-Assembly. J. Phys. Chem. B 2006, 110, 2798-2803.

9. Liu, H.L.; Yang, Z.L.; Meng, L.Y.; Sun, Y.D.; Wang, J.; Yang, L.B.; Liu, J.H.; Tian, Z.Q. Three-Dimensional and Time-Ordered Surface-Enhanced Raman Scattering Hotspot Matrix. J. Am. Chem. Soc. 2014, 136, 5332-5341. [CrossRef]

10. Huang, T.; Cao, L.; Zhang, X.; Xiong, X.Y.; Xu, J.J.; Xiao, R.S. A facile method to fabricate a novel 3D porous silicon/gold architecture for surface enhanced Raman scattering. J. Alloys Compounds 2019, 790, 127-133. [CrossRef]

11. Fu, F.Y.; Yang, B.B.; Hu, X.M.; Tang, H.Y.; Zhang, Y.P.; Xu, X.Y.; Zhang, Y.Y.; Touhid, S.S.B.; Liu, X.D.; Zhu, Y.F.; et al. Biomimetic synthesis of 3D Au-decorated chitosan nanocomposite for sensitive and reliable SERS detection. Chem. Eng. J. 2020, 392, 123693. [CrossRef]

12. Huang, Y.P.; Huang, S.C.; Wang, X.J.; Bodappa, N.; Li, C.Y.; Yin, H.; Su, H.S.; Meng, M.; Zhang, H.; Ren, B.; et al. Shell-Isolated Tip-Enhanced Raman and Fluorescence Spectroscopy. Angew. Chem.-Int. Ed. 2018, 57, 7523-7527. [CrossRef]

13. Zrimsek, A.B.; Chiang, N.H.; Mattei, M.; Zaleski, S.; McAnally, M.O.; Chapman, C.T.; Henry, A.I.; Schatz, G.C.; Van Duyne, R.P. Single-Molecule Chemistry with Surface- and Tip-Enhanced Raman Spectroscopy. Chem. Rev. 2017, 117, 7583-7613. [CrossRef] [PubMed]

14. Verma, P. Tip-Enhanced Raman Spectroscopy: Technique and Recent Advances. Chem. Rev. 2017, 117, 6447-6466. [CrossRef] [PubMed]

15. van Schrojenstein Lantman, E.M.; Deckert-Gaudig, T.; Mank, A.J.G.; Deckert, V.; Weckhuysen, B.M. Catalytic processes monitored at the nanoscale with tip-enhanced Raman spectroscopy. Nat. Nanotechnol. 2012, 7, 583-586. [CrossRef]

16. Li, J.F.; Huang, Y.F.; Ding, Y.; Yang, Z.L.; Li, S.B.; Zhou, X.S.; Fan, F.R.; Zhang, W.; Zhou, Z.Y.; Wu, D.Y.; et al. Shell-isolated nanoparticle-enhanced Raman spectroscopy. Nature 2010, 464, 392-395. [CrossRef] [PubMed]

17. Xie, L.; Lu, J.; Liu, T.; Chen, G.; Liu, G.; Ren, B.; Tian, Z. Key Role of Direct Adsorption on SERS Sensitivity: Synergistic Effect among Target, Aggregating Agent, and Surface with Au or Ag Colloid as Surface-Enhanced Raman Spectroscopy Substrate. J. Phys. Chem. Lett. 2020, 11, 1022-1029. [CrossRef]

18. Bailey, M.R.; Martin, R.S.; Schultz, Z.D. Role of Surface Adsorption in the Surface-Enhanced Raman Scattering and Electrochemical Detection of Neurotransmitters. J. Phys. Chem. C 2016, 120, 20624-20633. [CrossRef]

19. Skoupa, V.; Jenistova, A.; Setnicka, V.; Matejka, P. Role of $\mathrm{TiO}_{2}$ Nanoparticles and UV Irradiation in the Enhancement of SERS Spectra To Improve Levamisole and Cocaine Detection on Au Substrates. Langmuir 2019, 35, 4540-4547. [CrossRef] 
20. Hai, L.T.; Tiggelaar, R.M.; Berenschot, E.; van den Berg, A.; Tas, N.; Eijkel, J.C.T. Postdeposition UV-Ozone Treatment: An Enabling Technique to Enhance the Direct Adhesion of Gold Thin Films to Oxidized Silicon. ACS Nano 2019, 13, 6782-6789.

21. Chatterjee, A.; Gale, D.J.G.; Grebennikov, D.; Whelan, L.D.; Merschrod, S.E. Surface potential and morphology mapping to investigate analyte adsorption effects on surface enhanced Raman scattering (SERS). Chem. Commun. 2017, 53, 12024-12027. [CrossRef]

22. Zhang, H.; Yang, H.; Shentu, B.Q. Effect of surface modification of titanium dioxide on the UV-C aging behavior of silicone rubber. J. Appl. Polym. Sci. 2019, 136, 47170. [CrossRef]

23. Yanez-Pacios, A.J.; Martin-Martinez, J.M. Surface modification and adhesion of wood-plastic composite (WPC) treated with UV/ozone. Compos. Interfaces 2018, 25, 127-149. [CrossRef]

24. Verkuijlen, R.O.F.; van Dongen, M.H.A.; Stevens, A.A.E.; van Geldrop, J.; Bernards, J.P.C. Surface modification of polycarbonate and polyethylene naphtalate foils by UV-ozone treatment and mu Plasma printing. Appl. Surf. Sci. 2014, 290, 381-387. [CrossRef]

25. Murakami, T.N.; Fukushima, Y.; Hirano, Y.; Tokuoka, Y.; Takahashi, M.; Kawashima, N. Surface modification of polystyrene and poly(methyl methacrylate) by active oxygen treatment. Colloids Surf. B-Biointerfaces 2003, 29, 171-179. [CrossRef]

26. Zeng, Y.; Du, X.; Hou, W.; Liu, X.J.; Zhu, C.; Gao, B.B.; Sun, L.D.; Li, Q.W.; Liao, J.L.; Levkin, P.A.; et al. UV-Triggered Polydopamine Secondary Modification: Fast Deposition and Removal of Metal Nanoparticles. Adv. Funct. Mater. 2019, 29, 1901875. [CrossRef]

27. Kudryashov, S.I.; Nastulyavichus, A.A.; Tolordava, E.R.; Kirichenko, A.N.; Saraeva, I.N.; Rudenko, A.A.; Romanova, Y.M.; Panarin, A.Y.; Ionin, A.A.; Itina, T.E. Surface-Enhanced IR-Absorption Microscopy of Staphylococcus aureus Bacteria on Bactericidal Nanostructured Si Surfaces. Molecules 2019, $24,4488$. [CrossRef] [PubMed]

28. Gao, M.; Lin, X.; Li, Z.; Wang, X.; Qiao, Y.; Zhao, H.; Zhang, J.; Wang, L. Fabrication of highly sensitive and reproducible 3D surface-enhanced Raman spectroscopy substrates through in situ cleaning and layer-by-layer assembly of Au@Ag nanocube monolayer film. Nanotechnology 2019, 30, 345604. [CrossRef]

29. Kumar, S.; Lodhi, D.K.; Singh, J.P. Highly sensitive multifunctional recyclable Ag-TiO2 nanorod SERS substrates for photocatalytic degradation and detection of dye molecules. RSC Adv. 2016, 6, 45120-45126. [CrossRef]

30. Saliba, N.; Parker, D.H.; Koel, B.E. Adsorption of oxygen on Au (111) by exposure to ozone. Surf. Sci. 1998, 410, 270-282. [CrossRef]

31. Sun, K.J.; Kohyama, M.; Tanaka, S.; Takeda, S. Theoretical Study of Atomic Oxygen on Gold Surface by Huckel Theory and DFT Calculations. J. Phys. Chem. A 2012, 116, 9568-9573. [CrossRef] [PubMed]

32. Lee, D.J.; Kim, D.Y. Hydrophobic Paper-Based SERS Sensor Using Gold Nanoparticles Arranged on Graphene Oxide Flakes. Sensors 2019, 19, 5471. [CrossRef] [PubMed]

33. Zhang, Y.; Liu, R.J.; Ma, X.F.; Liu, X.Y.; Zhang, Y.X.; Zhang, J. Ag nanoparticle decorated MnO2 flakes as flexible SERS substrates for rhodamine 6G detection. RSC Adv. 2018, 8, 37750-37756. [CrossRef]

34. Yang, L.; Hu, J.; He, L.; Tang, J.; Zhou, Y.; Li, J.; Ding, K. One-pot synthesis of multifunctional magnetic $\mathrm{N}$-doped graphene composite for SERS detection, adsorption separation and photocatalytic degradation of Rhodamine 6G. Chem. Eng. J. 2017, 327, 694-704. [CrossRef]

35. Eckertová, L. Mechanism of Film Formation. In Physics of Thin Films; Springer: Boston, MA, USA, 1977; pp. 72-114.

36. Worley, C.G.; Linton, R.W. Removing sulfur from gold using ultraviolet/ozone cleaning. J. Vac. Sci. Technol. A 1995, 13, 2281-2284. [CrossRef]

37. Moldovan, A.; Feldmann, F.; Krugel, G.; Zimmer, M.; Rentsch, J.; Hermle, M.; Roth-Fölsch, A.; Kaufmann, K.; Hagendorf, C. Simple Cleaning and Conditioning of Silicon Surfaces with UV/Ozone Sources. Energy Procedia 2014, 55, 834-844. [CrossRef]

38. Vig, J.R. UV/Ozone Cleaning of Surfaces: A Review. In Surface Contamination; Mittal, K.L., Ed.; Springer: Boston, MA, USA, 1979; pp. 235-254.

(C) 2020 by the authors. Licensee MDPI, Basel, Switzerland. This article is an open access article distributed under the terms and conditions of the Creative Commons Attribution (CC BY) license (http://creativecommons.org/licenses/by/4.0/). 